\title{
NUEVOS APORTES SOBRE LA CARTOGRAFÍA DE LA COSTA GALLEGA Y LA DEFENSA DEL IMPERIO A FINALES DEL SIGLO XVIII
}

Data recepción: 2012/01/15

Data aceptación: 2012/06/13

Pedro Luengo Gutiérrez

Contacto autor: pedroluengo@us.es

Universidad de Sevilla

\section{RESUMEN}

Recientemente se ha localizado en El Puerto de Santa María una colección de mapas fechados en torno a 1768 entre los que se encuentran representados distintos enclaves gallegos como Camariñas, la Ría de Vigo, la de Bares, Corcubión y finalmente la Rada de Lagos, ya en el Algarve portugués. Junto a estos puertos se han identificado otros americanos y asiáticos, al parecer realizados por la misma mano. Por un lado, suponen un aporte documental al desarrollo de las defensas de estos puntos en un momento de posible ataque británico. Por otra parte, la colección en conjunto parece apuntar como trasfondo al conflicto entre España y Francia con Gran Bretaña a escala global. En este sentido las colecciones compiladas por las Escuelas de Navegación debieron servir de predecesoras de los futuros depósitos hidrográficos que nacerían a escala europea poco más tarde.

Palabras clave: Gran Bretaña, cartografía, Escuelas de navegación, Historia Atlántica

\section{ABSTRACT}

A collection of maps dating to around 1768 was recently found in the town of El Puerto de Santa Maria. It comprised representations of Galician coastal locations such as Camarinas and Corcubion, the sea estuaries (rías) of Vigo and Bares, and also the Rada de Lagos in the Portuguese Algarve. Maps of American and Asian ports were discovered along with them and seem to have been drawn by the same hand. As well providing documentary evidence of the development of the defences of these areas at a time when there was a likelihood of attack by the British, the collection also appears to provide a backdrop to the global war that pitted Spain and France against Great Britain. In this respect, the collections compiled by navigation schools would have served as the predecessors of the future hydrographic offices that would start appearing across Europe a little later.

Keywords: Great Britain, cartography, navigation schools, Atlantic history

La Guerra de los Nueve años (1739-1748) tuvo a Galicia como un punto geoestratégico de los enfrentamientos entre Gran Bretaña y España, situación que continuó durante los conflictos posteriores hasta la Paz de París (1764). Su posición permitía advertir con la mayor celeridad a la Flota de Indias y a los territorios americanos de una posible ofensiva. Además, facilitaba el conocimiento de los contactos de la metrópoli británica con Gibraltar y con otros enclaves de su dilatado imperio colonial. Todo esto implicó la necesidad de un mejor conocimiento de puertos naturales como Vigo, Corcubión, Pontevedra y Muros. Muchos de ellos, muy valorados por sus características orográficas, carecían de los sistemas defensivos suficientes. Su interés militar durante la segunda mitad del siglo XVIII queda reflejado en las fuentes gráficas conservadas'. El ingeniero Francisco Llobet, en su Descripción de las Plazas y Puestos Fortificados del Reino de Galicia, de sus costas y fronteras[...] con noticia de las proyectadas y de las aprobadas (1757), ya ponía de manifiesto que no existía en aquel reino puesto de suficiente envergadura para afrontar 
una invasión ${ }^{2}$. Consecuentemente desde los primeros años de la segunda mitad del siglo se había iniciado la construcción de sistemas defensivos en poblaciones como La Coruña, Corcubión o Camariñas, entre otras ${ }^{3}$. Además de levantar estructuras militares era importante conocer las posibilidades de calado de las rías, para lo que se comisionó el sondeo de algunas de ellas a Miguel Marín en $1754^{4}$. El renovado conocimiento de las costas gallegas se centró en Pontevedra, Tuy y La Coruña, pero incluyó también algunas de las rías de mayor valor militar, entre las que destacan Camariñas o Corcubión ${ }^{5}$. Estos trabajos vinieron a sustituir a los realizados en 1739 y 1740 en los que las fortificaciones no se habían representado 6 .

Poco más tarde, en la publicación de Jacques Nicolas Bellin, los principales puertos gallegos serían expuestos al conocimiento europeo ${ }^{7}$. Enclaves como La Coruña, Ferrol, Vigo o Corcubión aparecen en la publicación francesa ofreciendo algunos sondeos y olvidando algunos de los proyectos de fortificación realizados. Desde el impacto internacional que supuso la primera edición del Bellin los ingenieros españoles comenzaron a perfeccionar aquellos enclaves peor representados. Aunque en los mapas sobre Galicia no se hace referencia explícita, es probable que las deficientes representaciones francesas obligaran a ampliar las mediciones y a incorporar los renovados elementos defensivos.

Por parte española, tras las iniciativas de mediados de siglo habría que esperar hasta los años ochenta para iniciar otro gran proyecto. En este caso al gaditano Vicente Tofiño de San Miguel le fue encomendado levantar un Atlas marítimo de España, donde los puertos gallegos tenían una lógica y amplia representación, llegando hasta un tercio del total de planos ${ }^{8}$. Aquí se incluyen representaciones de la Ría del Barquero (con el número 28), las rías de Ferrol, Coruña y Betanzos (29), la de Ferrol (30), la de Corcubión y Pontevedra (31) y la de Vigo hasta Camariñas (32). A esto habría que añadir una vista de las costas de Galicia (39). Desde 1739 hasta esta edición de 1789 las pautas evolutivas son claras. En primer lugar la atención se diversifica, no focalizando exclusivamente el interés en los puertos tradicionales sino dotando a la Marina de información práctica en caso de ataque. En segundo lugar se multiplican los datos sobre sondeos evidenciando una preocupación por el calado de los buques. Por último las fortificaciones, realizadas o proyectadas, cobran especial significado. Cualquiera de los tres elementos ofrecía una evidente ventaja ante cualquier ataque extranjero, pues tal vez solo contaría con los datos poco profundos de la mencionada obra de Bellin.

Junto a estas publicaciones, fruto de grandes empresas cartográficas, habría que incorporar otros informes vinculados a problemas concretos devenidos de las relaciones diplomáticas, especialmente los relacionados con la guerra con Gran Bretaña. Todas las potencias europeas aprovecharon los recursos publicados para enriquecer los conocimientos adquiridos por sus propias expediciones. Esto se convertía en un arma fundamental en distintos conflictos armados que afectaban por igual a los territorios europeos que a los asiáticos o a los americanos. De esta forma comenzaron a crearse instituciones cuyo objetivo era acumular información sobre un territorio cada día más extenso y diverso.

En este contexto característico del último tercio del siglo XVIII hay que entender la colección conservada actualmente en El Puerto de Santa María, compuesta por un total de diecinueve mapas de puertos localizados en Galicia, Portugal, América y Asia ${ }^{9}$. La colección fue dada a conocer por Cardoso y Becerra en 1992, aunque desgraciadamente no suscitó el interés que la serie merecía ${ }^{10}$. Más recientemente aquellos mapas más claramente emparentados con la expedición de Juan de Cassens a Asia han sido reciente objeto de estudio ${ }^{11}$. Más allá de todo esto, parece aceptado que todos los mapas comparten pautas de representación que hacen pensar en una datación similar y probablemente en un mismo autor. Solo dos de ellos están fechados, en 1768 (Malaca) y 1769 (Malvinas), mientras que otro, el que representa Lucepara, está vinculado con la citada expedición del Buen Consejo comandada por Juan de Cassens ${ }^{12}$. Otros ejemplos pueden fecharse gracias a elementos incorporados, normalmente estructuras defensivas, como ocurre en el caso de La Habana. El mapa apunta el proyecto de construcción del Fuerte de San Carlos de la Cabaña, por lo que parece evidente que debió 
realizarse en los años inmediatos a la devolución de la capital cubana a la corona española13.

En resumen, aquellos mapas que pueden datarse por uno u otro medio apuntan al final de la década de los sesenta. Se trataría en cualquier caso de la fecha de realización in situ y no del momento de elaboración de una posible copia posterior. La unidad gráfica que presentan la mayoría de los mapas hace pensar que fueron realizados por un mismo autor. Esta posibilidad puede corresponder a dos hipótesis diversas. En primer lugar puede pensarse que se trata de la producción propia de algún ingeniero militar que conservara este material como parte de sus méritos en el cuerpo. En este caso debería tratarse de un profesional que tras trabajar en los sondeos de las costas gallegas, se enrolara en el Buen Consejo junto a Juan de Cassens rumbo a Asia y además participara en el reconocimiento de costas americanas desde Cuba hasta las Malvinas. Hasta el momento no se ha localizado ningún dibujante que cumpla el requisito de haber trabajado en destinos tan dispares en la segunda mitad del siglo XVIII. Una segunda opción, que resulta más verosímil a la luz de la documentación actual, es que se trate de una serie de copias de una colección conservada en alguno de los departamentos de la Real Escuela de Guardamarinas de la península, preferiblemente Cádiz, aunque sin descartar la de Ferrol.

La mayoría de los mapas conocidos con referencia explícita a las Escuelas de Navegación tienen una datación tardía ya a partir de 1770, cuando no solo habían abierto en los tres departamentos la Escuela de Navegación, sino también la Academia de Guardamarinas. Solo Cádiz contó al parecer con una colección más antigua, recolectada probablemente desde los inicios de su fundación en 1717. En contra de la posibilidad de que la colección de El Puerto de Santa María tenga alguna relación con la gaditana se encuentra que ningún mapa ha sido marcado con el escudo de la institución. En cambio en otros ejemplos conservados procedentes de la Escuela sí que se incorpora.

Una tercera característica que unifica por completo la colección y que puede estar detrás de una copia posterior es el miedo a la amenaza británica. Manila y La Habana habían sido to- madas en 1762 y la colonia de Sacramento (Río de la Plata) se había entregado a cambio junto con La Florida al año siguiente. Las Malvinas a finales de la década de los sesenta se encuentran disputadas por franceses, españoles y de nuevo británicos ${ }^{14}$. El resto de representaciones, tales como Batavia, Puerto Rico, Montevideo o Maldonado pueden fácilmente explicarse como objetivos probables de las tropas británicas en los alrededores de los enclaves citados. De hecho muchos de ellos caerían tras las ofensivas inglesas de principios del siglo XIX. En este caso no sería ya la Guerra de los Nueve Años, causa de la reforma de las defensas de algunos puertos gallegos, sino la Guerra de los Siete Años, la que provocaría una revisión de las características de los puertos.

La datación de todos los diecinueve planos a finales del siglo XVIII parece clara, pero puede apuntarse una fecha antesquem en 1784. A la hora de realizar los mapas generales de Galicia por parte de Tomás López en este momento se cita expresamente la larga nómina de fuentes utilizadas entre las que se detallan representaciones no solo de Corcubión, Camariñas, Barquero, Vivero, Vigo y Bayona, que podrían corresponder con todas las aquí estudiadas, sino además Ferrol, Betanzos y La Coruña ${ }^{15}$ (Fig. 1).

Mucho después de su realización la colección pasaría a formar parte del Depósito Hidrográfico donde se encontrarían a principios del siglo XX. Allí serían seleccionadas para formar parte de la Exposición Iberoamericana de Sevilla en 1929.

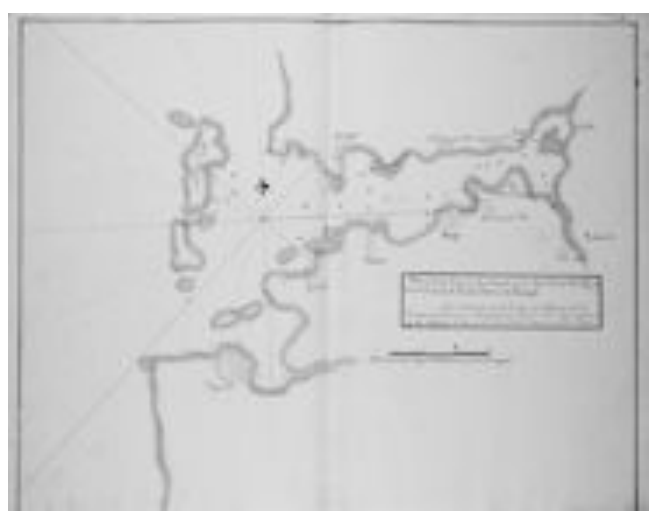

Fig. 1. Ría de Vigo. AMEPSM, leg. 1634, Papeles Antiguos, tomo 46 , apéndices, $n^{\circ} 17$ 
Es con motivo de su exhibición cuando el grupo queda relegado y se incorpora a su ubicación actual en el Archivo Municipal de El Puerto de Santa María ${ }^{16}$. Desgraciadamente hoy ha perdido cualquier contexto documental en el que probablemente estaría inscrito, haciendo más difícil las conexiones con otras fuentes de archivo. A pesar de ello, este grupo de diecinueve mapas ejemplifica el grado de mundialización que la corona española debía afrontar ante el continuo envite británico. Como ocurría en Francia o en Gran Bretaña, los repositorios de imágenes se hacían indispensables, ya que el correcto conocimiento del terreno gracias a este tipo de trabajos de reconocimiento facilitaba la toma de decisiones en la metrópoli a la hora de actuar a nivel global. Así se fueron creando distintas Oficinas Hidrográficas, la primera en Francia en 1720, el Dêpot des Plans, Cartes et Journaux de la Marine, o el Hydrographic Office, creado en Gran Bretaña en 1795. Solo dos años más tarde se fundaría el Depósito Hidrográfico español.

En aquellas potencias marítimas la adquisición y conservación de material cartográfico precedió a la creación de la institución. Algo similar ocurrió en España, que supo aprovechar no solo la labor de los ingenieros militares sino también la reforma que la Marina vivió a principios de siglo. En 1717 se crearía la Academia de Guardamarinas en Cádiz ${ }^{17}$. Poco más tarde, en 1749, se crea la Escuela de Pilotos en los tres departamentos: Cádiz, Ferrol y Cartagena. Su función era proveer de pilotos a la marina de guerra, más allá de servir de formación a la flota mercante. Aunque el principal objetivo de la primera institución era la formación de oficiales de la armada, pronto acumuló una colección de mapas y planos que debía ser importante. Habría que esperar hasta 1776 para que los otros dos departamentos, Ferrol y Cartagena, contaran con su propia Academia de Guardamarinas.

Aunque las tres Escuelas de Navegación dedicaron sus esfuerzos a almacenar cartas náuticas de las expediciones más recientes, parece ser Cádiz la que obtuvo una mejor colección, no solo de Andalucía y América sino también de territorios asiáticos y otros enclaves europeos, principalmente italianos y griegos. Cádiz debió acumular una colección envidiable que hoy día está disgregada entre muchas instituciones $^{18}$. Su vinculación con América explica que fuera la receptora de mapas de Nueva España y otros territorios del Virreinato del Perú, además de las cartas de la expedición de Juan de Cassens a Asia. En contra de lo que podría pensarse, territorios como Galicia o Portugal también eran accesibles en Cádiz, así como territorios más alejados como el Mar Negro ${ }^{19}$. De entre los responsables de muchas de estas copias habría que destacar a Pedro Rivelles, quien debió llegar a Cádiz procedente de la institución de Cartagena. De la Escuela de este Departamento hay menos información, aunque parece destacable el trabajo de Francisco Cervantes, continuador de la labor de Rivelles ${ }^{20}$. En Ferrol, por último, habrá que esperar a los trabajos de Andrés de la Cuesta, correspondientes a distintos puertos gallegos o asturianos además de las Malvinas o Río de la Plata²1.

La situación bélica que vivió Galicia en el siglo XVIII obligó a considerar su defensa de una forma más general y como consecuencia de ello se entiende más fácilmente la labor cartográfica iniciada tempranamente en la Escuela del Ferrol. Quizás los enclaves principales en un primer momento eran las plazas fortificadas de La Coruña, Vigo y Ferrol, que además estaban apoyadas por los buques de los Arsenales. Además habría que añadir en el litoral Ribadeo y Cedeira en el norte; Camariñas, Finisterre, Corcubión y Cée, en la Costa de la Muerte; Marín, y Bayona en las Rías Bajas; y finalmente La Guardia, Goyán, Amorín, Tuy y Salvatierra en la frontera con Portugal22.

De toda esta problemática el autor de los mapas conservados en el Puerto de Santa María hizo una selección de enclaves, quizás de segunda categoría en algunos casos, pero muy útiles para afrontar un posible ataque marítimo desde el norte. De entre todos cabe destacar los de Corcubión, Camariñas y Vigo. El de la Ría de Vivero es interesante por tratarse de un caso poco representado, mientras que la portuguesa Rada de Lagos resulta reveladora en este contexto global, quizás como solución a un ataque a la Flota de Indias camino de Cádiz. Para plantear mejor las particularidades e información que ofrecen cada una de las vistas se tratarán de forma consecutiva. 
El mapa de la Ría de Corcubión es probablemente el que más datos aporta para datar la colección en su conjunto (Fig. 2). En primer lugar el núcleo poblacional de Corcubión es considerablemente más grande que el representado décadas atrás por Desnaux, apareciendo ya con las dimensiones que plantearía Tofiño. Más interés aún suponen las fortificaciones ${ }^{23}$. Para Corcubión se proyectó un fuerte en el extremo de su bahía conocido con el nombre de Santa Cruz, que al menos en 1755 seguía sin construirse. Esta batería es citada en los planos de Llobet como "del Agro". Frente al Fuerte de Santa Cruz, en la otra orilla se proyectó la Batería de la Punta Farnelo, o según Llobet "del Espino". Ambas darían una mejor protección a la inmediata ciudad, pero probablemente se quedaron en meras pretensiones. El mismo año de 1739 se proyectan las obras del Castillo del Cardenal, en Olveira, y en la orilla contraria, en A Ameixenda, el del Príncipe ${ }^{24}$. La solución es exactamente la misma pero más cerca del mar. El fuego cruzado desde ambas baterías, además de la supuesta cadena que los unía, protegía a la ciudad y su puerto. Las obras en cambio no comenzarían hasta 1744, dejando en un segundo plano las obras en el Fuerte de Santa Cruz y en la Punta Farnelo ${ }^{25}$. Como apuntan algunos autores la construcción del Castillo del Príncipe y del Cardenal fue dirigida por Carlos Lemaur, junto con el delineador Antonio Exarch, apareciendo dicha construcción reflejada poco después en las obras de Francisco Llobet ${ }^{26}$.

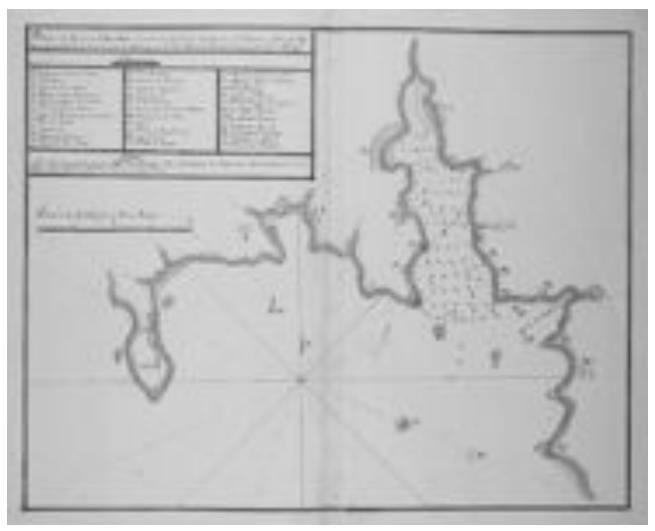

Fig. 2. Ría de Corcubión. AMEPSM, leg. 1634, Papeles Antiguos, tomo 46, apéndices, $n^{\circ} 5$.
En el mapa de El Puerto de Santa María aparece además de la batería del Príncipe y del Cardenal, una tercera estructura defensiva en el Cabo de Cee, correspondiente con lo que en otros documentos se titula la Batería de Santa Cruz. Como acaba de mencionarse Francisco Llobet había diseñado en 1755 cuatro baterías que servirían para defender toda la embocadura de la ría, pero ninguna de ellas corresponde con ésta ${ }^{27}$. Las dos que actualmente se conservan, la del Cardenal y la del Príncipe, se comenzaron en 1744 pero el proceso constructivo se prolongaría, mientras que las otras dos, al parecer, no llegaron a iniciarse. En resumen, puede pensarse que la representación hoy conservada en Cádiz supone un paso posterior, en el que tras observar que la construcción de la pareja de baterías más cercana a Corcubión no aportaría capacidad defensiva, mientras que una batería en el Cabo de Cee sería de gran ayuda a la batería de San Carlos en Finisterre.

La vinculación entre esta imagen y la producción de la Escuela del Departamento de Cádiz es difícil de probar pero afortunadamente la Biblioteca del Congreso en Washington conserva un mapa de la Ría de Corcubión de los años centrales del siglo XVIII procedente de la institución española ${ }^{28}$. Este ejemplar no ofrece información sobre el estado de sus defensas pero sí sobre los sondeos de la Ría, mediciones que no coinciden con el caso conservado en la colección gaditana.

Además de las estructuras defensivas los mapas a veces resaltan algunos elementos arquitectónicos que servirían de referencias en el horizonte. En este ejemplo concreto se trata de un pequeño dibujo del perfil de la iglesia de Santa María das Areas en Finisterre. Una diferencia destacable entre la representación del dibujo y el edificio conservado hoy es la configuración de la torre, mucho más esbelta en el mapa. Es posible que se trate de una simplificación del propio dibujante, que no es especialmente detallista en este sentido. Corcubión y la fortificación de su ría suponen un punto de gran interés en la Galicia del siglo XVIII, como complemento ineludible en la defensa de Finisterre. Algo similar puede decirse de la Ría de Camariñas, alejada del problema concreto de Ferrol y La Coruña. Tanto Corcubión como Camariñas tenían muchas ven- 
tajas ante un posible ataque extranjero, alejando además el problema de otros centros más relevantes como La Coruña o Vigo (Fig. 3).

Probablemente la base de la fortificación de la ría de Camariñas se deba a La Ferriere, o al menos a un planteamiento de 1740 que quedó suspendido una década ${ }^{29}$. Finalmente las obras se desarrollarían a partir de los planos de Francisco Llobet en 1755. Se construiría el conocido como Castillo del Soberano, en la Punta del Boy. Su configuración es distinta a la de Corcubión ofreciendo una solución más adaptada a las características de los navíos de guerra del siglo XVIII. Junto con el del Soberano, Miguel Marín proyectó una rectificación de un proyecto de Llobet para construir la batería de Merexo. Finalmente se desestimó su construcción. En cambio, el plan defensivo que elaborara también Llobet fue ampliado en 1753. Se planteó la construcción de dos nuevos elementos defensivos, uno en la Punta de Barreira y otro en la de La Ínsula, aunque ninguno de ellos llegó a terminarse ${ }^{30}$. El mapa que se trata, conservado en la colección gaditana, no hace referencia a estas medidas defensivas, ya que como ocurre en otros casos lo más probable es que su construcción hubiera sido desestimada.

Al igual que en el caso de Corcubión, la colección de la Escuela del Departamento de Cádiz contaba con al menos una representación de la Ría de Camariñas, conservada hoy en Washington $^{31}$. También aquí las referencias a los proyectos defensivos han sido desestimadas, mientras

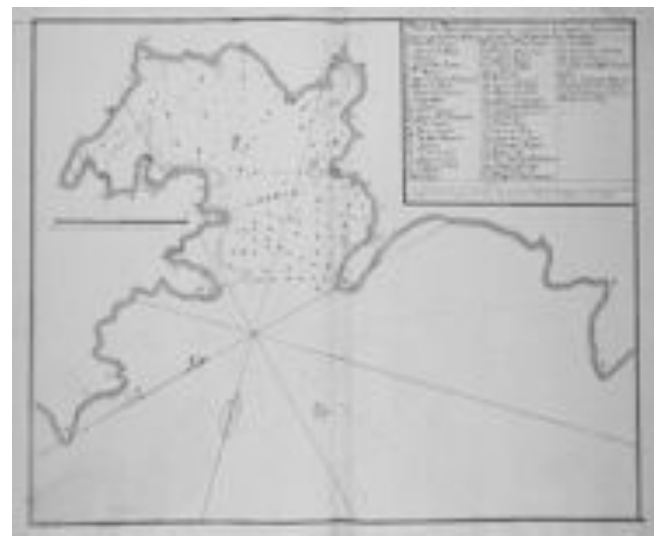

Fig. 3. Ría de Camariñas. AMEPSM, leg. 1634, Papeles Antiguos, tomo 46 , apéndices, $n^{\circ} 8$. que los sondeos no coinciden completamente. Muy probablemente se trate de dos obras fruto de expediciones cercanas en el tiempo pero inconexas.

Camariñas se encuentra a mitad de camino entre Corcubión y Ferrol, pero la importancia de los Arsenales y de una ciudad como La Coruña en el siglo XVIII obligaba a valorar otras opciones siguiendo la costa hacia Asturias ${ }^{32}$. Así es fácil comprender el interés de Ferrol en hacerse con mapas de puertos como Ribadeo y en este caso concreto de la Ría de Vivero, zona que apenas había suscitado el interés de los cartógrafos precedentes. En general, la desembocadura del río Landrove no recibió tanto interés por la corona durante el siglo XVIII. Hasta el momento, distintos estudios apuntaban a la representación de Tofiño como la primera carta marina realizada de la zona ${ }^{33}$. Probablemente el mapa de El Puerto de Santa María sea un estado anterior de la misma. Desgraciadamente la escasez de estructuras defensivas levantadas durante el siglo XVIII hace difícil precisar la datación exacta, que debe compartir por otra parte con el resto de las obras. La estructura militar que defendía Vivero estaba formada por una muralla muy mal conservada a finales del siglo y una serie de puertas y una fortaleza realizados en buena parte en el siglo XVI.

Con la de Vivero se abordaba una posibilidad de resguardarse en la costa norte, y con ella los alrededores de Ferrol, pero Vigo también destacaba en este momento como enclave de la costa gallega. El ataque anglo-holandés a Vigo de 1702 puso a prueba las posibilidades defensivas de sus estructuras, desde el Castillo del Castro hasta la pareja del Castillo de Corbeiro y de Rande. Pasado el ataque enemigo se plantearon diversas posibilidades que permitieran encarar con garantías la defensa de la plaza ${ }^{34}$. A mediados del siglo, aún se debatía entre las propuestas de Fray Martín Sarmiento y José Cornide en la que las parejas de baterías, en uno u otro emplazamiento, suponían la clave del éxito. En el plano que pertenece a la colección gaditana subsiste el Castillo de Rande, pero el de Corbeiro ha sido sustituido por una Batería. En resumen parece claro que ninguna de estas propuestas está prevista en los mapas de El Puerto. En cambio sí parece vislumbrarse lo que será el proyecto de 
Cayetano Paveto de 1788, quien planteará una batería en Corbeira y una reforma de Rande. En cambio las baterías de Arroas y Teis no aparecen representadas.

Como ocurre en los ejemplares estudiados las fechas de realización deben estar rondando los años finales de la década de los sesenta o los primeros de la siguiente. No responden a las iniciativas de los años centrales del siglo, y mucho menos a los planteamientos de la década de los ochenta en adelante. Esto que ocurre con los casos gallegos es perfectamente trasladable al resto de ejemplares de la colección, tanto los correspondientes a puertos asiáticos como a los americanos.

Hasta aquí los mapas de puertos gallegos conservados hoy en el archivo de El Puerto de Santa María. Pero además de estos cuatro, a los americanos y asiáticos, hay que añadir una representación de la Rada de Lagos (Portugal). La representación no debió ser la única realizada en este momento, ya que en el Museo Naval madrileño se cuenta con otra versión ${ }^{35}$. De una u otra forma, Lagos ofrecía un resguardo natural escasamente fortificado pero altamente interesante para los navíos que llegaban al Cabo de San Vicente. El problema de esta playa era la facilidad para encallar, por lo que cualquier información sobre el sondeo era de vital importancia. Seguramente el empuje de Gibraltar como eje comercial del imperio británico y el traslado del comercio americano a Cádiz hizo pensar en la necesidad de contar con buenas mediciones sobre posibles puertos donde resguardar buques de cierto calado.

Además de las particularidades de cada uno de los planos, la colección ofrece algunos rasgos que la unifican y que deben ser planteados de forma conjunta. Un primer aspecto que debe ser analizado es la selección de puertos dentro de la costa gallega. Parece previsible encontrarse en estos momentos con representaciones de la Ría de Vigo, de Corcubión o incluso de Camariñas. Mucho más extraño resulta la incorporación de sondeos de la Ría de Bares o de la portuguesa de Lagos (Figs. 4 y 5). De la misma forma parece sorprendente que no se tuvieran en cuenta otros mapas de La Coruña o de Ferrol, puertos muy desarrollados. Una explicación que puede estar detrás de la selección puede ser de nuevo un posible ataque británico. Corcubión era un puerto natural que había visto mejoradas sus medidas defensivas, pero la experiencia de mediados de siglo había demostrado que también era necesario conocer otros puertos naturales que pudieran servir de resguardo a la flota. Para ello no resultaba indispensable que estuvieran perfectamente fortificados mientras que el conocimiento exacto de los sondeos era vital. Con este carácter de puerto de resguardo es fácil entender los sondeos de la Ría de Bares, carente de elementos defensivos y poco utilizados en el trasiego de grandes buques en este momento. Las notables ausencias de algunos puertos, que pueden explicarse por una pérdida dentro de la misma colección, pueden apoyar la relación con los conflictos armados expuesta. Un ejemplo notable es el de La Coruña, puerto ultramarino en ciernes desde el reinado de Felipe $V$, que no apa-

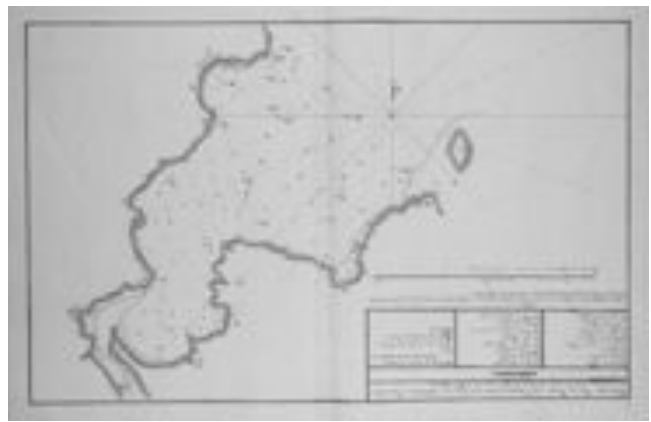

Fig. 4. Ría de Bares o del Barquero. AMEPSM, leg. 1634, Papeles Antiguos, tomo 46, apéndices, $n^{\circ} 6$.

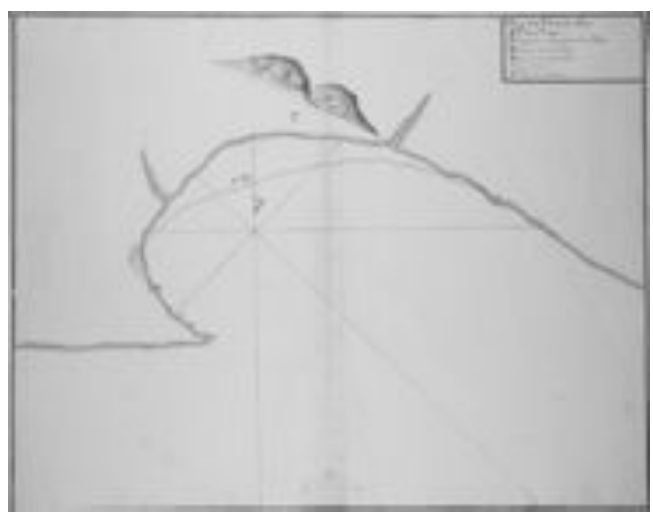

Figura 5. Rada de Lagos. AMEPSM, leg. 1634, Papeles Antiguos, tomo 46 , apéndices, $n^{\circ} 4$. 
rece entre los mapas de la colección gaditana. La Coruña, aunque ofrecía un indudable recurso militar, estaba potenciando su perfil mercantil y burgués ${ }^{36}$.

La importancia de dicha ciudad en la historia atlántica durante la segunda mitad del siglo XVIII ha sido puesta ya de manifiesto por otros estu$\operatorname{dios}^{37}$. Pero La Coruña, además de un conglomerado defensivo unido con El Ferrol, requería de otros puertos secundarios que reforzaran sus posibilidades ante un eventual ataque. Es así como se comprenden con mayor facilidad los distintos mapas presentados, incorporándose a la problemática más global de un Atlántico con un desarrollo particular en este momento histórico. De hecho, si se acepta la relación de la colección con el conflicto borbónico-británico de la segunda mitad del siglo XVIII, las consecuencias del mismo hay que entenderlas fundamentalmente dentro de un marco de Historia Atlántica con innegables consecuencias en el devenir colonial de las Indias Orientales, dando lugar a un problema global. Como han apuntado ya distintos autores, tanto desde la historia comparada como desde otras perspectivas, el Atlántico en este momento puede estudiarse como un área acuciada por particulares circuitos mercantiles, militares y culturales. Esta colección gaditana subraya cómo las políticas de defensa desde la corte madrileña iban comprendiendo el problema británico en toda la extensión del Atlántico. Al mismo tiempo que se buscaba la posibilidad de reparar los daños producidos por el Tratado de París con un ataque al imperio inglés, se trabajaba en mejorar los puntos geoestratégicos desamparados tanto en la Península como en América y Asia.

Las costas gallegas, en toda su extensión, tenían un lógico papel en la Historia Atlántica. El movimiento de buques británicos, más allá del propio contacto con Francia, hacía de Galicia un enclave destacado en la defensa de la península y del resto de territorios ultramarinos. Aunque la Escuela de Navegación de Ferrol no tuviera hasta lo que se conoce la colección cartográfica de la de Cádiz, la importancia del territorio gallego queda demostrada por el número de representaciones conservadas. De entre todas estas habría que destacar la colección de El Puerto de Santa María, no ya por la información propia de los mapas, sino sobre todo por encontrarse en un contexto donde el problema global de la monarquía hispana y los conflictos internacionales están claramente presentes.

Esta colección gaditana, cuya parte referente a Galicia ha sido abordada en este estudio, permite comparar las actuaciones en pos de la defensa de sus territorios emprendidas por dos imperios en ocaso ${ }^{38}$. Desde la India y las Filipinas hasta las Trece Colonias y el Virreinato de Río de la Plata, se notaron las réplicas del Tratado de París en forma de medidas fiscales y consecuentes revueltas. La relación entre la metrópoli y las posesiones ultramarinas debía cambiar ineludiblemente y para ello el conocimiento cartográfico y las políticas de construcción de sistemas defensivos se situaron entre las primeras medidas abordadas desde Londres y Madrid. 


\section{NOTAS}

${ }^{1}$ El elevado número de mapas ha despertado el interés de los investigadores. Entre otros estudios se puede destacar: Soraluce Blond, José Ramón. Castillos y fortificaciones de Galicia. La arquitectura militar de los siglos XVI-XVIII. La Coruña: Fundación Pedro Barrié de la Maza, 1985. Más recientemente y como muestra del ingente material existente y de las posibilidades de interpretación que ofrece debe destacarse Vigo Trasancos, Alfredo (dir.), Sánchez García, Jesús Ángel y Taín Guzmán, Miguel (coord.). Planos y dibujos de arquitectura y urbanismo. Galicia en los siglos XVI y XVII. Santiago de Compostela: Consello Galego de Colexios de Aparelladores e Arquitectos Técnicos, 2003.

${ }^{2}$ Capel, Horacio (et. al). Los ingenieros militares en España, siglo XVIII: repertorio biográfico e inventario de su labor cientifica y espacial. Barcelona: Universidad de Barcelona, 1983. p. 256.

${ }^{3}$ Francisco Llobet desarrolló desde muy temprano una amplia actividad como ingeniero en Galicia que culminaría con la elaboración de un plano de la muralla de Ferrol de 1769. Capel, Horacio (et. al). op. cit.

4 Pueden destacarse los sondeos de Corcubión, Cartoteca Centro Geográfico del Ejército (CCGE) Ar.E-T.4C.5-165; o de Camariñas, CCGE, AR.ET.4-C.5-160.

${ }^{5}$ Capel, Horacio. "Los ingenieros militares y el sistema de fortificación en el siglo XVIII" en Cámara Muñoz, Alicia. Los ingenieros militares de la monarquía hispánica en los siglos XVII y XVIII. Madrid: Ministerio de Defensa, 2005.

${ }^{6}$ Estos trabajos anteriores correspondientes a Camariñas y Corcubión se conservan en el Archivo General de Simancas (AGS), Sección Mapas, Planos y Dibujos (MPD), 30, 056 y AGS, MPD, 30, 052 respectivamente.

7 Bellin, Jacques Nicolas. Le Petit Atlas Maritime. Paris: 1764

${ }^{8}$ Tofiño de San Miguel, Vicente. Atlas marítimo de España. Madrid: Dirección de Hidrografía, 1789. Museo Naval (MN). A-10055.

${ }^{9}$ Archivo Municipal del Puerto de Santa María (AMEPSM), Leg 1634, Papeles Antiguos, tomo 46, apéndices.
${ }^{10}$ Cardoso Alcántara, Teo y Becerra Fabra, Ana. "La ruta comercial directa Cádiz-Manila: aportación cartográfica a su estudio". Cádiz e Iberoamérica. Cádiz: Diputación de Cádiz, 1992.

11 Luengo, Pedro. "Cádiz-Manila. Cartografía de los viajes de Juan de Casens". López Guzmán, Rafael (coord.). Andalucía en América. Arte y Patrimonio. Granada, Atrio, 2012, pp. 171-192.

${ }^{12}$ La datación de la representación del archipiélago solo un año antes de la Crisis de las Malvinas en 1770 hace pensar en el contenido militar, especialmente vinculado al conflicto internacional contemporáneo, de toda la colección.

${ }^{13}$ El Fuerte de San Carlos fue levantado siguiendo el proyecto de Silvestre Abarca finalizándose en 1774.

${ }^{14}$ De las Malvinas y de Río de la Plata se hicieron copias más tardías en el Escuela del Departamento de Ferrol, apuntando al interés de estos enclaves en Galicia. Cfr. Malvinas. Biblioteca del Museo Naval (BMN), MN-48-D-7. Río de la Plata. BMN, MN-46-B-1.

${ }^{15}$ López Gómez, Antonio y Manso Porto, Carmen. Cartografía del siglo XVIII. Tomás López en la Real Academia de la Historia. Madrid: Real Academia de la Historia, 2006. P. 45.

${ }^{16}$ Cardoso Alcántara, Teo y Becerra Fabra, Ana. Op. cit.

${ }^{17}$ Lafuente, Antonio y Peset, José Luis. "Las Academias militares y la inversión en ciencia en la España ilustrada (1750-1760)" en Acta Hispanica ad Medicinae Scientiarumque Historiam Illustrandam. Vol. 2. 1982, pp. 193209.

${ }^{18}$ Buena parte del fondo se conserva en la Biblioteca del Congreso en Washington, aunque la Biblioteca Nacional de España también conserva restos.

${ }^{19}$ Del ámbito gallego podría destacarse el Plano de las tres rías de Ferrol, Betanzos y de La Coruña, realizado en Cádiz a partir de copia hecha en Ferrol en 1759. MR /42/550.

20 Rivelles realizó en Cartagena un mapa de Palma. Alejo Berlinguero y Nicolás Berlinguero en cambio elaborarían mapas de Denia, Génova, Marsella, o la propia Cartagena.

${ }^{21}$ A los casos americanos citados deben añadirse distintos mapas de la costa de Brasil, hoy en la Biblioteca del Museo Naval.

${ }^{22}$ Rodríguez-Villasante Prieto, Juan A. op. cit. p. 28

${ }^{23}$ Tradicionalmente se ha hablado de tres estructuras defensivas: la del Cardenal, la del Príncipe y la de San Carlos. A estas se añaden las proyectadas en Punta de Espino y Do Agro. Además de estas la documentación permite pensar que se barajaron otras opciones. Rodríguez-Villasante Prieto, Juan A. Historia y tipología arquitectónica de las defensas de Galicia. Funcionalidad, forma y ejecución del diseño clasicista. La Coruña: Sada, 1984. P. 108.

${ }^{24}$ Puede verse el plano y proyecto de la batería de la punta de Cantelo y de la de Camboa en Archivo General de Simancas, AGS, MPD, 30, 054. El mismo año se proyecta una tercera en la Punta del Fornelo representada en AGS, MPD, 30, 053. Existe por último un último perfil de la Batería de la Punta de Cantelo obra de Carlos Desnaux conservada en AGS, MPD, 30, 055.

${ }^{25}$ La batería sería representada por Francisco Llobet en varias ocasiones De 1751 se conservan en la Cartoteca del Centro Geográfico del Ejército (CCGE). Ar.E-T.4-C.5-156 o Ar.E-T.4C.5-162. Dos años más tarde la batería es presupuestada y comenzada por el Cuerpo de Ingenieros como consta en el plano y perfil de la misma CCGE, Ar.E-T.4-C.5-163 y Ar.E-T.4-C.5-164. Solo un año más tarde se envía a Miguel Marín a realizar el sondeo, conservado en CCGE, Ar.E-T.4-C.5-165. En 1757 pueden destacarse el Plano de la Batería del Cardenal de la Ría de Corcubión. Cartoteca del Archivo General Militar de Madrid. C-17/4. CCGE. Ar.ET.4-C.5-166. También de mediados del siglo XVIII es el mapa conservado hoy en la Sección de Mapas de la Biblioteca del Congreso en Washington, LC LusoHispanic World, 788.

${ }^{26}$ Lemaur, tras haber estudiado en la Real Escuela de Matemáticas de París con Belidor y desarrollar una intensa carrera militar en Europa fue convencido por el Embajador de España en París, Pignateli, para incorporarse como ingeniero a la corona española a partir de 1750. Soraluce Blond, José Ramón. Op. cit. p. 148.

27 "Plano de la Ría o Puerto de Corcubión [...] en él se demuestra la 
posición de las cuatro baterías propuestas para su defensa". CAGMM. C-17/1. A las del Cardenal y del Príncipe habría que unir la de la Punta del Espino y la de la Punta del Agro, proyectadas respectivamente en CAGMM C $-17 / 2$ y $\mathrm{C}-17 / 3$.

${ }^{28}$ Biblioteca del Congreso en Washington, Sección de Mapas, LC LusoHispanic World, 788.

${ }^{29}$ Los restos de la batería permanecieron hasta 1944, cuando la construcción del nuevo puerto de Camariñas lo hizo desaparecer. Rodríguez-Villasante Prieto, Juan A. op. cit. p. 108. Véase también Soraluce Blond, José Ramón. Op. cit. pp. 144-146.

30 íbid. Pp. 144.
${ }^{31}$ Biblioteca del Congreso en Washington, Sección de Mapas, LC LusoHispanic World, 793.

32 Sobre La Coruña en este momento puede ampliarse en Vigo Trasancos, Alfredo. A Coruña y el siglo de las luces: la construcción de una Ciudad de Comercio (1700-1808). Santiago de Compostela: Universidad, Servicio de Publicaciones e Intercambio Científico; La Coruña: Universidad, Servicio de Publicaciones, 2007.

33 Soraluce Blond, José Ramón. Op. cit. p. 114.

34 íbid. pp. 173-175.

35 Plano de la Bahía de Lagos. Biblioteca del Museo Naval. MNMN-117-6.
${ }^{36}$ Vigo Trasancos, Alfredo. A Coruña y el siglo de las luces: la construcción de una Ciudad de Comercio (1700-1808). Santiago de Compostela: Universidad, Servicio de Publicaciones e Intercambio Científico; La Coruña: Universidad, Servicio de Publicaciones, 2007.

${ }^{37}$ O'Flanagan, Patrick. Port cities of Atlantic Iberia, c. 1500-1900. Aldershot: Ashgate, 2008.pp. 207-230.

${ }^{38}$ Elliot, John. España, Europa y el mundo de Ultramar (1500-1800). Madrid: Taurus, 2010. P. 277-278. 